1. Fakharzadeh, S.S., and Kazazian, H.H., Jr. 2000. Correlation between factor VIII genotype and inhibitor development in hemophilia A. Semin. Thromb. Hemost. 26:167-171.

2. Dickson, P., et al. 2008. Immune tolerance improves the efficacy of enzyme replacement therapy in canine mucopolysaccharidosis I. J. Clin. Invest. 118:2868-2876.

3. Barton, N.W., et al. 1991. Replacement therapy for inherited enzyme deficiency - macrophagetargeted glucocerebrosidase for Gaucher's disease. N. Engl. J. Med. 324:1464-1470.

4. Starzyk, K., Richards, S., Yee, J., Smith, S.E., and Kingma, W. 2007. The long-term international safety experience of imiglucerase therapy for Gaucher disease. Mol. Genet. Metab. 90:157-163.

5. Rohrbach, M., and Clarke, J.T. 2007. Treatment of lysosomal storage disorders: progress with enzyme replacement therapy. Drugs. 67:2697-2716.

6. Kakkis, E.D., et al. 2001. Enzyme-replacement therapy in mucopolysaccharidosis I. N. Engl.J. Med. 344:182-188

7. Wraith, J.E., et al. 2007 Enzyme replacement therapy in patients who have mucopolysaccharidosis I and are younger than 5 years: results of a multinational study of recombinant human alpha-L-iduronidase (laronidase). Pediatrics. 120:e37-e46.

8. Shull, R.M., et al. 1994. Enzyme replacement in a canine model of Hurler syndrome. Proc. Natl. Acad. Sci. U. S. A. 91:12937-12941.

9. Kakkis, E.D., et al. 1996. Long-term and high-dose trials of enzyme replacement therapy in the canine model of mucopolysaccharidosis I. Biochem. Molec. Med. 58:156-167.

10. Kakkis, E., et al. 2004. Successful induction of immune tolerance to enzyme replacement therapy in canine mucopolysaccharidosis I. Proc. Natl. Acad. Sci. U. S. A. 101:829-834.

11. Gao, W., et al. 2007. Contrasting effects of cyclosporine and rapamycin in de novo generation of alloantigen-specific regulatory T cells. Am. J. Transplant. 7:1722-1732.

12. Lim, D.G., et al. 2007. Effect of immunosuppressants on the expansion and function of naturally occurring regulatory $\mathrm{T}$ cells. Transpl. Immunol. 18:94-100.

13. Adams, A.B., Pearson, T.C., and Larsen, C.P. 2001. Conventional immunosuppression and co-stimulation blockade. Philos. Trans. R. Soc. Lond. B Biol. Sci. 356:703-705.

14. San Segundo, D., Fábrega, E., López-Hoyos, M., and Pons, F. 2007. Reduced numbers of blood natural regulatory $T$ cells in stable liver transplant recipients with high levels of calcineurin inhibitors. Transplant Proc. 39:2290-2292.

15. Tiede, I., et al. CD28-dependent Rac1 activation is the molecular target of azathioprine in primary human CD4+ T lymphocytes. J. Clin. Invest. 111:1133-1145

16. Joseph, A., Munroe, K., Housman, M., Garman, R., and Richards, S. 2008. Immune tolerance induction to enzyme-replacement therapy by co-administration of short-term, low-dose methotrexate in a murine Pompe disease model. Clin. Exp. Immunol. 152:138-146.

17. Turner, C.T., Hopwood, J.J., Bond, C.S., and Brooks, D.A. 1999. Immune response to enzyme replacement therapy: 4-sulfatase epitope reactivity of plasma antibodies from MPS VI cats. Mol. Genet. Metab. 67:194-205.

18. Sun, B., Bird, A., Young, S.P., Kishnani, P.S., Chen, Y.T., and Koeberl, D.D. 2007. Enhanced response to enzyme replacement therapy in Pompe disease after the induction of immune tolerance. Am. J. Hum. Genet. 81:1042-1049.

19. Kishnani, P.S., et al. 2007. Recombinant human acid [alpha]-glucosidase: major clinical benefits in infantile-onset Pompe disease. Neurology. 68:99-109.

20. Ohashi, T., Iizuka, S., Ida, H., and Eto, Y. 2008. Reduced alpha-Gal A enzyme activity in Fabry fibroblast cells and Fabry mice tissues induced by serum from antibody positive patients with Fabry disease. Mol. Genet. Metab. 94:313-318.

\title{
It takes two to tango: cigarette smoke partners with viruses to promote emphysema
}

\author{
Rubin M. Tuder and Jeong H. Yun
}

Program in Translational Lung Research, Division of Pulmonary Sciences and Critical Care Medicine, Department of Medicine, University of Colorado Denver School of Medicine, Denver, Colorado, USA.

\begin{abstract}
Viruses constitute a constant and renewed threat to humans. Not only do viruses cause disease directly due to their tissue tropism and pathogenicity, but they have also been linked to autoimmunity. In their study in this issue of the JCI, Kang et al. show that exposure to cigarette smoke induces alterations in the innate immune response to viral infection and that these changes hasten alveolar destruction characteristic of emphysema in mice (see the related article beginning on page 2771). This study builds on evidence that patients with chronic obstructive pulmonary disease have clinical exacerbations associated with viral or bacterial infections, which lead to worsened lung function and increased mortality. This novel paradigm may aid related genetic, biomarker, and therapeutic developments and provides important insights into the pathogenesis of emphysematous lung destruction.
\end{abstract}

The worldwide magnitude of diseases caused by cigarette smoking has outweighed

Nonstandard abbreviations used: COPD, chronic obstructive pulmonary disease; dsRNA, double-stranded RNA; eIF2 $\alpha$, eukaryotic initiation factor- $\alpha$; MAVS, mitochondrial antiviral signaling; PAMP, pathogenassociated molecular pattern; PKR, RNA-dependent protein kinase; poly(I:C), polyinosinedeoxycytidylic acid; RIG-1, retinoic acid-inducible gene-I; RLH, RIG-1-like helicase.

Conflict of interest: The authors have declared that no conflict of interest exists.

Citation for this article: J. Clin. Invest. 118:2689-2693 (2008). doi:10.1172/JCI36536. our ability to combat them or even slow their relentless expansion. Cigarette smoke is the major cause of chronic obstructive pulmonary disease (COPD), which is characterized by chronic cough due to excessive mucus production (chronic bronchitis) and/or alveolar destruction leading to increased airspaces, known as centrilobular emphysema. COPD is the only major disease whose contribution to morbidity and mortality continues to increase, potentially displacing stroke as the third major worldwide cause of mortality by 2020 (1). This stark reality forces us to recognize the limitations of current disease paradigms and to search for answers that go beyond the traditional ways of thinking about COPD.

The classic paradigm of cigarette smokeinduced COPD has proposed that the alveolar destruction and enlargement is a direct consequence of inflammation and the associated imbalance in the extracellular matrix protease and antiprotease response, which leads to degradation of the elastin alveolar framework. This hypothesis continues to be revisited, as it has not led to significant therapeutic advances against the disease. Recent hypotheses have emphasized the resemblance of lung injury caused by cigarette smoke to the effects of aging in the lung, which results from the interaction between environmental stresses and homeostatic molecular and cellular processes involved in organismal protection (2). Cigarette smoke may thus co-opt some of the molecular signaling pathways involved in cellular sensing of environmental stresses, such as those triggered by starvation, radiation, or hypoxia, leading to progressive disruption 


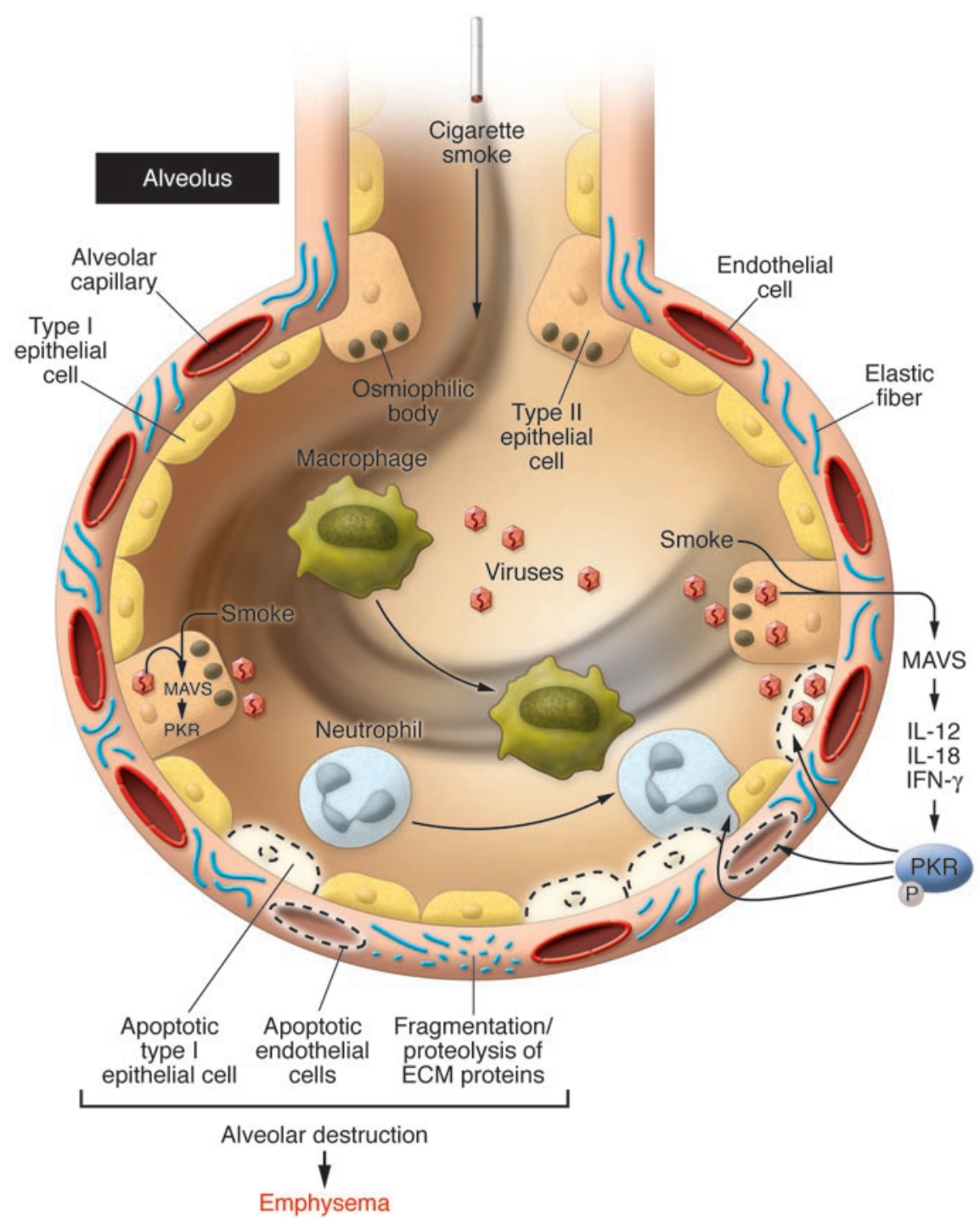

\section{Figure 1}

Synergistic interaction between cigarette smoke and RNA viruses in emphysema in mice. The combination of exposure to cigarette smoke and RNA viruses leads to alveolar cell apoptosis involving type I epithelial, type II epithelial, and endothelial cells, and enhanced alveolar inflammation (with influx of neutrophils and macrophages). As shown by Kang et al. (5) in their study in this issue of the $\mathrm{JCl}$, the process of alveolar destruction is set up via activation of the RLH adaptor protein MAVS, the cytokines IL-12, IL-18, and IFN- $\gamma$, and the phosphorylation of the kinase PKR (5). Furthermore, this process may course with an imbalance between matrix protease and antiproteases, favoring fragmentation of extracellular matrix proteins, including elastin. The cell signaling pathways triggered in alveolar epithelial cells infected by RNA viruses or synthetic dsRNA are represented in Figure 2. of organ maintenance, with the undesirable activation of apoptotic and inflammatory responses that characterize the alveolar destruction observed in emphysema (3). Indeed, oxidative stress is involved in both cigarette smoke and aging (4). Notwithstanding that innate immune responses have evolved to shield against bacterial, parasitic, and viral infections - the main evolutionary forces driving adaptation of uni- and multicellular organisms - cellular stress signaling may interface with innate immunity in the promotion of disease caused by exposure to cigarette smoke.

In their current study in this issue of the JCI, Kang et al. report the results of a series of elegant experiments using wild-type and knockout mice and reveal how cigarette smoke induces alterations in innate responses triggered by double-stranded RNA (dsRNA) or influenza virus, resulting in accel- erated alveolar destruction (5) (Figure 1). The finding that this synergism relies on a molecular pathway involving mitochondrial antiviral signaling (MAVS) protein, IL-18, IL-12, IFN- $\gamma$, and the RNA-dependent protein kinase (PKR) (Figure 1) reveals molecular facets not previously recognized with respect to how cigarette smoke may injure the lung and promote emphysema. This novel paradigm may aid related genetic, biomarker, and therapeutic developments and, importantly, provides insights that may shed light on old and present disease concepts, including the role of inflammation and alveolar cell apoptosis in emphysematous lung destruction (6).

\section{Cigarette smoke partners with RNA viruses}

Epidemiological evidence supports the concept that COPD patients are at increased risk of viral and bacterial infections, caus- ing significant worsening of COPD-related symptoms, such as cough, fever, and mucus production (also known as clinical exacerbations), often requiring hospitalization. These COPD exacerbations are often followed by subsequent clinical deterioration, documented as significant decrements of forced expiratory volume at 1 second (a measure used to diagnose the stage of COPD) and increased mortality (7). The mechanisms underlying the interaction of innate immunity caused by viral or bacterial infections with cigarette smoke in the pathogenesis of COPD remain unknown. Elucidation of these mechanisms may offer insights into how viral or bacterial infections may accelerate the airway and/or parenchymal injury that characterizes COPD. Innate immune responses rely vastly on the recognition of specific pathogen-associated molecular patterns (PAMPs) by TLRs, triggering 


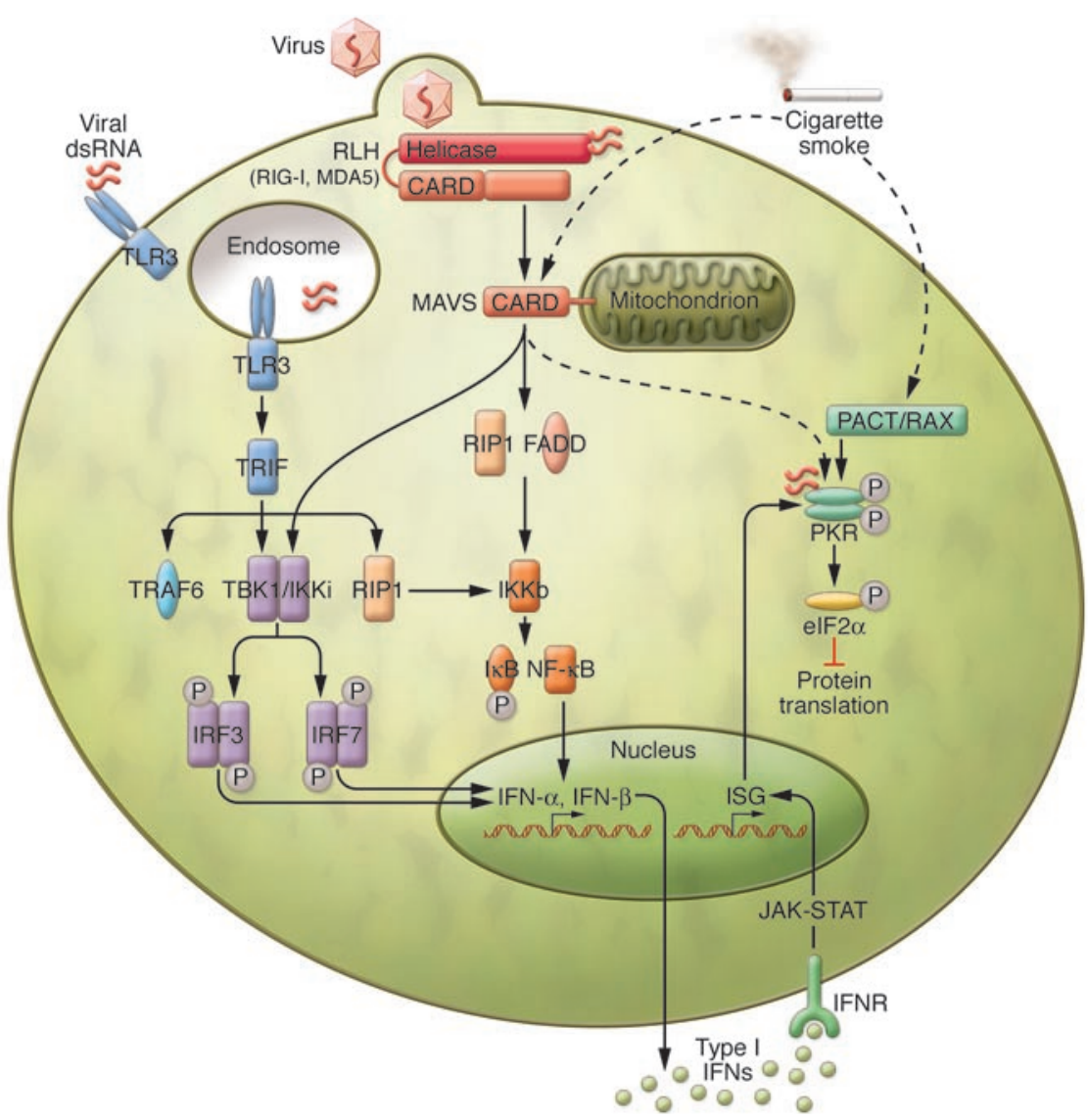

Figure 2

Type I IFN production and PKR activation triggered by TLR3 or the RLH system by natural or synthetic dsRNA. Activation of TLR3 by viral dsRNA leads to the recruitment of Toll/IL-1R homology domain-containing adaptor-inducing IFN- $\gamma$ (TRIF), which activates TNF receptorassociated factor 6 (TRAF6), TANK-binding kinase 1/lkB kinase i (TBK1/IKKi), and receptorinteracting protein 1 (RIP1). TBK1 phosphorylates the IFN regulatory factors (IRFs) 3 and 7. Cytoplasmic dsRNA is also recognized by the RLH system, which interacts with MAVS via caspase recruitment domains (CARDs). MAVS recruits IKK and TBK1, and this converges with TLR3 signaling and leads to NF- $\kappa B$ activation and the induction of type I IFNs (e.g., INF- $\alpha$ and INF- $\beta$ ). MAVS also activates NF-KB through Fas-associated death domain-containing protein (FADD) and RIP1 interaction. The binding of type I IFNs to their receptor (IFNR) causes JAK/ STAT-mediated synthesis of IFN-stimulated gene (ISG) products, including PKR. Although it is unclear whether PKR may be directly stimulated by the interaction of MAVS and dsRNA (as indicated by the dashed arrow), the endogenous protein PACT/RAX can also activate PKR independently of dsRNA. As suggested by the results of the current study by Kang et al. (5), cigarette smoke may (as reflected by dashed arrows) affect lung cell responses to RNA viruses by enhancing MAVS-PKR signaling and therefore trigger alveolar cell death. Figure modified with permission from Nature Immunology (9) and Cell Research (12).

inflammatory cell responses that converge and result in NF-KB- or IFN-dependent gene transcription (Figure 2). Among the TLRs $3,7,8$, and 9 , which recognize viral PAMPs, TLR3 binds to viral dsRNA or its synthetic homolog polyinosinedeoxycytidylic acid $[\mathrm{poly}(\mathrm{I}: \mathrm{C})]$ presented on the cell surface or in endosomal compartments (8). Viral PAMPs are also detected by a parallel cytosolic recognition system involving retinoic acid-inducible gene-1 (RIG-1) and melanoma differentiation-associated pro- tein-5 (MDA5), also known as RIG-1-like helicases (RLHs) (9) (Figure 2). dsRNAs from actively replicating viruses bind to the $\mathrm{C}$ terminus region of RLH and contribute to the building of antiviral defenses. This mode of TLR3-independent signaling is the main innate response involved in the interaction between synthetic or viral dsRNA and cigarette smoke, as Kang et al. demonstrate that TLR3-null mice show minimal improvement in lung inflammation, cytokine production, and alveolar enlargement when compared with wild-type littermates (5). Moreover, Kang et al. unravel the contribution of the signaling pathway downstream of members of the RNA helicase family, which requires the adaptor molecule MAVS and PKR to mediate the lung effects of the interaction between dsRNA and cigarette smoke in lung inflammation and alveolar enlargement (Figures 1 and 2). Importantly, this system has broader cellular functions since it is directly involved in molecular responses triggered by organismal stresses (10), as highlighted below.

\section{Role of MAVS and PKR in inflammation and alveolar cell apoptosis}

The data reported in this issue by Kang et al. position MAVS as a critical determinant of alveolar cell destruction caused by the combination of exposure to cigarette smoke and poly $(\mathrm{I}: \mathrm{C})$ or influenza infection, since MAVS-knockout mice are protected against cigarette smoke and poly(I:C)or influenza virus-induced alveolar cell apoptosis and inflammatory cell influx (5). MAVS is a mitochondrial adapter protein linking dsRNA recognition by RIG-1 or MDA5 to downstream NF-KB activation and IFN production (Figure 2) (11).

PKR is required for alveolar destruction and lies downstream of MAVS, IL-18, and IFN- $\gamma$ (5). PKR belongs to a family of virally triggered, stress-inducible genes that, with $2^{\prime}, 5^{\prime}$-oligoadenylate synthetase, account for cellular protective responses against viral infections (12). PKR activation and autophosphorylation require binding of dsRNA to a specific recognition domain within PKR. Although TLRs 4, 9, and 3 also activate PKR, NF-KB, and IFNs, Kang et al. (5) did not observe a synergistic interaction between cigarette smoke and a wide range of PAMPs, including activators of TLR4 (e.g., LPS) or TLR9 (e.g., CpG DNA). Given this apparent redundancy of TLR-triggered signaling, the specificity of the interaction of cigarette smoke with MAVS-dependent PKR activation is therefore surprising and in need of further investigation. PKR signaling leads to activation of NF- $\mathrm{KB}$ signaling, favoring inflammation and IFN production, and also to the phosphorylation of the transcription initiator eukaryotic initiation factor- $2 \alpha$ (eIF $2 \alpha)$.

Phosphorylation of eIF $2 \alpha$ is a central stress-response event that leads to inhibition of protein translation of several prosurvival proteins and selective synthesis of cell arrest/apoptosis proteins, such as 
activating transcription factor-3 (ATF-3) and ATF-4 (10). Indeed, PKR was shown to enhance apoptosis caused by TNF- $\alpha$ or LPS by activating ATF-3 or IFN regulatory factor-1 (IRF-1) (13). Kang et al. (5) provide tantalizing insights into the contribution of alveolar inflammation in relation to alveolar cell apoptosis in the alveolar destruction caused by cigarette smoke and dsRNA. Confirming their prior observation that alveolar cell apoptosis is required for experimental cigarette smoke-induced emphysema (14), they now demonstrate that the significant protection against cigarette smoke-induced emphysema observed in PKR-null mice also exposed to influenza virus correlates closely with a reduction in alveolar cell apoptosis, while inflammatory cell influx remains relatively unaffected when compared with that in the susceptible wild-type mice. The link between PKR activation and alveolar cell apoptosis may thus ultimately account for the extent of alveolar injury caused by the combination of exposure to cigarette smoke and activation of RLH-dependent signaling by viruses, such as influenza virus.

As aforementioned, PKR can also be activated by cellular stresses, including exposure to hydrogen peroxide, ceramide, arsenite, and thapsigargin (10). Furthermore, under conditions of stress, the cellular protein PKR-associated activator (PACT or RAX, for the human and mouse orthologs, respectively) can stimulate PKR independently of dsRNA (Figure 2). It is conceivable that oxidative stress caused by cigarette smoke alone has activated PKR in exposed lungs. The data reported by Kang et al. (5) suggest that cigarette smoke alone mildly increases PKR and eIF2 $\alpha$ phosphorylation, albeit to a lower level than that obtained when cigarette smoke exposure was associated with exposure to poly(I:C). Interestingly, poly(I:C) and influenza virus infection alone enhanced PKR and eIF $2 \alpha$ phosphorylation over baseline levels and resulted in a mild increase in lung inflammation, alveolar cell apoptosis, and airspace enlargement. In light of these findings, a central question that remains to be answered is how cigarette smoke can potentiate the lung responses caused by poly(I:C). This synergistic action does not appear to involve TLR4 or TLR4dependent signaling (including NF- $\kappa \mathrm{B}$ activation), which has been postulated to mediate some of the effects of cigarette smoke. One possibility is that cigarette smoke enhances poly(I:C) entrance into cells and/ or increases the recognition of dsRNA by the helicase system, since Kang et al. show a dosage effect of poly(I:C) on lung destruction by cigarette smoke and also show that PKR activation is dependent on MAVS. The complexity of this interaction is highlighted by the finding that in younger animals cigarette smoke may rather depress the IFN system, therefore increasing the risk of viral infections (15).

\section{Lessons learned from the mouse}

Modeling experimentally complex lung diseases imposes important limitations and significant challenges that may delay or misdirect translation of significant findings to humans. Human emphysema develops over decades of ongoing smoking or exposure to environmental pollutants (16), which cannot be accurately reflected by small animal modeling. The emphysematous phenotype caused by prolonged exposure (i.e., in excess of six months) to cigarette smoke is characteristically mild in mice and dependent on their genetic background (16). Accelerated emphysema has been documented in mice lacking the master regulator of antioxidant gene expression, NRF-2 (17) or the senescenceassociated protein-30 (18) and in mice with lung overexpression of IL-13 or IFN- $\gamma$ (19). In their current study, Kang et al. (5) demonstrate significant airspace enlargement and increased lung volumes within three weeks of exposure to the combination of cigarette smoke and poly(I:C). Is this speedy process of alveolar enlargement relevant to our understanding of the human disease? The destructive nature of this interaction may manifest only in more advanced stages of COPD in humans, as there is no present evidence that the interaction between cigarette smoke and viruses or bacterial infections early in the disease determines the rate of progression of airway or alveolar injury to the extent observed in this mouse model. This suggests that the disease has to progress through a set threshold to manifest the accelerated lung destruction in the setting of the interaction between cigarette smoke and viral infections. This threshold may be reached when the ongoing injury overcomes the lung's ability to maintain its cellular and molecular homeostasis, organ maintenance, or ability to repair (20). The rodent model may differ from the human disease, since several experimental and genetic manipulations result in an emphysematous phenotype in the rodent lung. This susceptibility may be inherent to the mouse lung's ongoing requirement of trophic signaling, which also imparts significant plasticity and ability to repair. It is conceivable that the activation of MAVS and PKR by cigarette smoke and dsRNA viruses may disrupt lung maintenance in the mouse at an accelerated pace, leading to emphysema in a relatively short time when compared with humans. Given our lack of understanding of the extent of similarities and differences in how rodent and human lungs respond to cigarette smoke-induced lung injury, the pathway outlined by Kang et al. merits subsequent studies aimed at translating this paradigm to COPD patients.

Recent medical practice has witnessed several examples of unanticipated side effects when centrally placed signaling molecules are targeted pharmacologically. This may indeed be the case with MAVS. MAVS appears to be required for protection against double-stranded viruses, as knockout mice have decreased type I IFN and NF- $\kappa \mathrm{B}$ responses and show increased mortality with disrupted viral clearance when compared with wild-type mice infected with vesicular stomatitis virus (11). Downregulation of MAVS and/or PKR may carry the significant risk of hampering an important antiviral protection system. Nonetheless, the finding that PKR-null mice can clear influenza virus as well as their wildtype littermates suggests that PKR may be a suitable target in COPD exacerbations.

The findings reported here by Kang et al. (5) offer a new window of opportunity for a better understanding of the unique interaction between cigarette smoke and innate immune responses. They reveal the potential importance of the interaction of cellular stress responses triggered by cigarette smoke with the innate immune response and highlight candidate molecules and signaling pathways to investigate in further studies.

\section{Acknowledgments}

This work was supported by NIH grants HL66554 and P50 HL084945 (Project 1), and Alpha 1 Foundation research grants (to R.M. Tuder).

Address correspondence to: Rubin M. Tuder, Program in Translational Lung Research, Division of Pulmonary Sciences and Critical Care Medicine, Department of Medicine, University of Colorado at Denver, School of Medicine, 4200 East 9th Avenue, Denver, BRB651, Colorado 
80262, USA. Phone: (303) 315-4475; Fax: (303) 315-5632; E-mail: Rubin.Tuder@ uchsc.edu.

1. Croxton, T.L., Weinmann, G.G., Senior, R.M. and Hoidal, J.R. 2002. Future research directions in chronic obstructive pulmonary disease. Am. J. Respir. Crit. Care Med. 165:838-844.

2. Tuder, R.M., Yoshida, T., Arap, W., Pasqualini, R., and Petrache, I. 2006. Cellular and molecular mechanisms of alveolar destruction in emphysema: an evolutionary perspective. Proc. Am. Thorac. Soc. 3:503-510.

3. Tuder, R.M., Yun, J.H., and Graham, B.B. 2008. Cigarette smoke triggers code red:p21CIP1/WAF1/ SDI1 switches on danger responses in the lung. Am. J. Respir. Cell Mol. Biol. 39:1-6.

4. Balaban, R.S., Nemoto, S., and Finkel, T. 2005. Mitochondria, oxidants, and aging. Cell. 120:483-495.

5. Kang, M.-J., et al. 2008. Cigarette smoke selectively enhances viral PAMP- and virus-induced pulmonary innate immune and remodeling responses in mice. J. Clin. Invest. 118:2771-2784.

6. Tuder, R.M., Petrache, I., Elias, J.A., Voelkel, N.F., and Henson, P.M. 2003. Apoptosis and emphysema: the missing link. Am. J. Respir. Cell Mol. Biol. 28:551-554.

7. Papi, A., et al. 2006. Infections and airway inflammation in chronic obstructive pulmonary disease severe exacerbations. Am. J. Respir. Crit. Care Med. 173:1114-1121.

8. Akira, S., Uematsu, S., and Takeuchi, O. 2006. Pathogen recognition and innate immunity. Cell. 124:783-801.

9. Kawai, T., and Akira, S. 2006. Innate immune recognition of viral infection. Nat. Immunol. 7:131-137.

10. Garcia, M.A., et al. 2006. Impact of protein kinase PKR in cell biology: from antiviral to antiproliferative action. Microbiol. Mol. Biol. Rev. 70:1032-1060.

11. Sun, Q., et al. 2006. The specific and essential role of MAVS in antiviral innate immune responses. Immunity. 24:633-642.

12. Seth, R.B., Sun, L., and Chen, Z.J. 2006. Antiviral innate immunity pathways. Cell Res. 16:141-147.

13. Der, S.D., Yang, Y.L., Weissmann, C., and Williams, B.R. 1997. A double-stranded RNA-activated protein kinase-dependent pathway mediating stressinduced apoptosis. Proc. Natl. Acad. Sci. U. S. A. 94:3279-3283.
14. Zheng, T., et al. 2005. Role of cathepsin S-dependent epithelial cell apoptosis in IFN- $\gamma$-induced alveolar remodeling and pulmonary emphysema. J. Immunol. 174:8106-8115.

15. Grath-Morrow, S., et al. 2008. Impaired lung homeostasis in neonatal mice exposed to cigarette smoke. Am. J. Respir. Cell Mol. Biol. 38:393-400.

16. Yoshida, T., and Tuder, R.M. 2007. Pathobiology of cigarette smoke-induced chronic obstructive pulmonary disease. Physiol. Rev. 87:1047-1082.

17. Rangasamy, T., et al. 2004. Genetic ablation of Nrf2 enhances susceptibility to cigarette smoke-induced emphysema in mice. J. Clin. Invest. 114:1248-1259.

18. Sato, T., et al. 2006. Senescence marker protein30 protects mice lungs from oxidative stress, aging, and smoking. Am. J. Respir. Crit. Care Med. 174:530-537.

19. Elias, J.A., Kang, M.J., Crouthers, K., Homer, R., and Lee, C.G. 2006. State of the art. Mechanistic heterogeneity in chronic obstructive pulmonary disease: insights from transgenic mice. Proc. Am. Thorac. Soc. 3:494-498.

20. Taraseviciene-Stewart, L., and Voelkel, N.F. 2008 Molecular pathogenesis of emphysema. J. Clin. Invest. 118:394-402

\title{
Lipid droplets: FSP27 knockout enhances their sizzle
}

\author{
Vishwajeet Puri and Michael P. Czech \\ Program in Molecular Medicine, University of Massachusetts Medical School, Worcester, Massachusetts, USA.
}

\begin{abstract}
Fat-specific protein of $27 \mathrm{kDa}$ (FSP27) is a highly expressed adipocyte protein that promotes triglyceride accumulation within lipid droplets. In this issue of the JCI, Nishino et al. show that FSP27 also helps to maintain the characteristically large unilocular lipid droplet structure within each white adipocyte (see the related article beginning on page 2808). Fragmentation of lipid droplets in white adipocytes from FSP27-KO mice caused both increased lipolysis and upregulation of genes enhancing mitochondrial oxidative metabolism. This increased energy expenditure in turn protected the mice from diet-induced obesity and insulin resistance. These new results highlight powerful mechanisms that tightly coordinate rates of triglyceride storage in lipid droplets with mitochondrial fatty acid oxidation in white adipocytes.
\end{abstract}

The handling and disposal of large volumes of greasy cooking ingredients like lard and vegetable oils is a messy business as any restaurant manager will lament. Human physiology faces the same daunting challenge for the efficient storage and metabolism of fats. To accomplish these feats, fat-storing cells, referred to as white

Nonstandard abbreviations used: CIDE, cell deathinducing DNA fragmentation factor 45-like effector; FSP27, fat-specific protein of $27 \mathrm{kDa}$; WAT, white adipose tissue.

Conflict of interest: The authors have declared that no conflict of interest exists.

Citation for this article: J. Clin. Invest. 118:2693-2696 (2008). doi:10.1172/JCI36554. fat cells or adipocytes, are uniquely specialized to accommodate over $95 \%$ of their cell volume as a single lipid droplet (Figure 1). Triglyceride within this unilocular lipid droplet can, in reaction to other signals, be hydrolyzed to fatty acids and glycerol, which move through the circulation to muscle and other tissues for energy production (fatty acids) and to liver for conversion to glucose (glycerol). Proteins are known to coat the surfaces of lipid droplets in adipocytes. However, until now, what molecules are required to maintain the distinctive unilocular characteristic of lipid droplets in white adipocytes has remained a mystery. In this issue of the JCI, Nishino et al.
(1) provide compelling evidence that one such protein is fat-specific protein of 27 $\mathrm{kDa}$ (FSP27; also known as Cidec), a highly and selectively expressed protein in human white adipocytes (2), recently found to colocalize with lipid droplets and regulate fat storage (3-5).

\section{Lipid droplets: composition and structure}

Lipid droplets within adipocytes are composed of a core of triglycerides and cholesteryl esters that are surrounded on their surfaces by a phospholipid monolayer associated with lipid droplet proteins (6). Perilipin was the first identified lipid droplet-specific protein that coats the surfaces of these structures in brown and white adipocytes and some steroidogenic cells (7). It is the prototypic member of a family of related proteins containing a "PAT" domain, so called for the members perilipin, a dipophilin (also known as adipose differentiation-related protein), and the tail-interacting protein of $47 \mathrm{kDa}$ (TIP47), but also includes the plasma membrane-associated protein S3-12 and myocardial lipid droplet protein/oxidative tissues-enriched PAT protein (MLDP/OXPAT) $(6,8)$. This domain, in 